\title{
COMPARATIVE ANALYSIS OF NUMERICAL SOLUTION OF OPTIMAL CONTROL PROBLEMS
}

\author{
Gulnaz Shangareeva $^{1}$, Igor Grigoryev ${ }^{2}$, Svetlana Mustafina ${ }^{3}$ \\ ${ }^{1,2,3}$ Sterlitamak Branch of the Bashkir State University \\ 37, Lenin Avenue, Sterlitamak city, 453103, RUSSIA
}

\begin{abstract}
In this article step by step algorithms were developed for solving optimal control problems based on the method of successive approximations and the method of variations in the space of controls. The algorithm of the method of successive approximations requires details of the problem to the boundary problem of the maximum principle. In turn, the algorithm of the variations is more versatile because it is based on iterating state variables and control in the phase space. A numerical study and comparative analysis of the developed algorithms performed at different values of accuracy.
\end{abstract}

AMS Subject Classification: 49M30, 65K05, 65K10

Key Words: method of successive approximations, the method of variations, optimal control, numerical algorithm

\section{Introduction}

Methods of the optimum control theory are intensively used in various application areas. At the moment the uniform universal method to solve this task is not developed that generally is explained by difficulty of problem of optimum control solving.

Numerical problem solving of optimum control is complicated by a number

Received: $\quad$ September 13, 2016

Revised: $\quad$ October 15, 2016

Published: November 15, 2016

(C) 2016 Academic Publications, Ltd. url: www.acadpubl.eu

${ }^{\S}$ Correspondence author 
of the various reasons. Let's list only the main of them: larger dimension of solvable tasks; existence of nondifferentiable functionals; existence of the difficult restrictions for management; existence of phase restrictions; possibility of appearance of many extremums. Due to a larger variety of statements of problems of optimum control and the specified difficulties there are many various approaches for their numerical decision now.

This work is devoted to an actual problem - development of efficient and universal algorithms of numerical problem solving of optimization.

\section{Problem Statement}

Consider the following optimal control

$$
\text { minimize } I(u)=G(x(T))
$$

subject to

$$
\begin{gathered}
\frac{d x_{i}}{d t}=f_{i}(t, x(t), u(t)), t \in\left[t_{0}, T\right], x(0)=x_{0}, \\
\phi(u) \leq 0
\end{gathered}
$$

where $u(t) \in R$ is the function characterizing the operating influence, $x(t) \in R^{n}$ is function describing a condition of process and $t$ is time.

Let's consider various algorithms for problem solving of optimal control.

\section{Method of Successive Approximations}

The problem of optimum control (1) - (3) by means of a maximum principle can be reduced to the solution of a boundary value problem of system of differential equations 2 n-go about.

Let's enter $n$ - dimensional vector $\psi=\left(\psi_{1}, \psi_{2}, \ldots, \psi_{n}\right)$ of conjugate variable and Hamilton function $H$ :

$$
H(t, x, \psi, u)=\psi^{T} f(t, x, u) .
$$

Let's write down the conjugated system:

$$
\frac{d \psi_{i}}{d t}=-\sum_{j=1}^{n} \frac{\partial f_{j}(t, x(t), u(t))}{\partial x_{i}}, i=1 \ldots n .
$$


with boundary conditions:

$$
\psi(T)=-\frac{\partial F(x(T))}{\partial x} .
$$

According to a maximum principle required optimum control delivers functions $H(t, x, \psi, u)$ at most $u \in U$ on at any $t \in\left[t_{0}, T\right]$, if $x(t)$ and $\psi(t)$ satisfy to system (1) and boundary conditions (6).

One of the most widespread methods of solution of the specified boundary value problem is the method of successive approximations in area of controls.

Let the approximation $u^{k}(t)$ be known. The next approximation $u^{k+1}(t)$ is made by the procedure including 3 stages:

1. Compute $x^{k}(t)$ by integrating system (2).

2. Calculate the value of variables $\psi^{k}(t)$ by using $x^{k}(t)$ and $u^{k}(t)$ in integration system (5) with starting conditions (6) in the opposite direction.

3. Define a new approximation $u^{k+1}(t)$ from a ratio (4), using values $x^{k}(t)$ and $u^{k}(t)$.

If the process of successive approximations converge, then we continue it until the subsequent approximations do not differ from each other within the specified accuracy. The received decision will satisfy to the maximum principle.

\section{Method of Variations}

In a method of variations on each iteration the variation $\delta u$ of control $u(t)$ is determined by minimization of linear part of an increment of the functionality $I(u)$, caused by this variation:

$\min _{\delta u \in \delta U} \delta I(d u)=\min _{\delta u \in \delta U} \int_{0}^{T} W_{0}^{T}(t) \delta u(t)$. The general scheme of a method of variations consists of 7 steps:

1. Set iterations number $k=0$ and guess an initial approximation of control $u^{k}(t) \in U$.

2. Compute $x^{k}(t)$ by solving (1).

3. Calculate $I^{k}(u)$ according to (3). Remember the value of criterion and control in sufficient number of points.

4. Calculate $W_{0}^{k}(t)=\frac{\delta I^{k}(u)}{\delta u}$ and determine $\delta U^{k}$. 
5. Find $\delta u^{k}$ of control $u^{k}(t)$ from

$$
\min _{\delta u \in \delta U^{k}} \delta I(d u)=\min _{\delta u \in \delta U^{k}} \int_{0}^{T}\left(W_{0}^{T}(t)\right)^{T} \delta u(t),
$$

6. Compute $u^{k+1}(t)=u^{k}(t)+\delta u^{k}$.

7. Go to step 2 until the condition $\delta u^{k}<\varepsilon$ is not satisfied.

\section{Discussion}

The software for the numerical calculations presented below in this article was developed in Borland Delphi environment. Further, errors will be calculated on Euclidean norm.

Example. Consider the following optimal control problem with the give initial conditions and restrictions:

$$
\begin{aligned}
& \text { Min: } I=x_{2}(2 \pi) ; \\
& \text { S to: }\left\{\begin{array}{l}
\dot{x_{1}}(t)=x_{2}(t), \\
\dot{x_{2}}(t)=-x_{1}(t)+u(t) ;
\end{array}\right. \\
& x_{1}(0)=0, x_{2}(0)=0 ;|u| \leq 1 ; t \in[0,2 \pi] .
\end{aligned}
$$

The necessary conditions, derived from calculus of variations, can be solved analytically; and we obtain for the state and control variables of the optimal trajectory:

$$
\begin{aligned}
& u^{*}(t)=\left\{\begin{array}{l}
-1, t \in\left[0, \frac{\pi}{2}\right], \\
1, t \in\left[\frac{\pi}{2}, \frac{3 \pi}{2}\right], \\
-1, t \in\left[\frac{3 \pi}{2}, 2 \pi\right] ;
\end{array}\right. \\
& x_{1}^{*}(t)=\left\{\begin{array}{l}
\cos t-1, t \in\left[0, \frac{\pi}{2}\right], \\
\cos t-2 \sin t+1, t \in\left[\frac{\pi}{2}, \frac{3 \pi}{2}\right], \\
\cos t-4 \sin t-1, t \in\left[\frac{3 \pi}{2}, 2 \pi\right] ;
\end{array}\right. \\
& x_{2}^{*}(t)=\left\{\begin{array}{l}
-\sin t, t \in\left[0, \frac{\pi}{2}\right], \\
-\sin t-2 \cos t, t \in\left[\frac{\pi}{2}, \frac{3 \pi}{2}\right], \\
-\sin t-4 \cos t, t \in\left[\frac{3 \pi}{2}, 2 \pi\right] .
\end{array}\right.
\end{aligned}
$$

The minimum value of functionality is equal to $x_{2}^{*}=(2 \pi)=-4$.

Table 1 shows the comparative analysis of results of the numerical solution of a test problem by method of a variation and the method of successive approximations in case of various values of initial approximation, accuracy of computation. 
Table 1: Simulation results for different initial guess and accuracy

\begin{tabular}{|c|c|c|c|c|}
\hline \multirow{2}{*}{ Options } & \multicolumn{2}{|c|}{$\varepsilon=10^{-3}$} & \multicolumn{2}{c|}{$\varepsilon=10^{-5}$} \\
\cline { 2 - 5 } & 1 & 2 & 1 & 2 \\
\hline$u_{0}$ & 0.9 & 0.9 & 0.1 & 0.1 \\
\hline Elasted time,c & 3.84 & 0.54 & 5.35 & 1.32 \\
\hline Error $\varepsilon_{u}$ & 2.962 & 0.998 & 2.00216 & 0,00998 \\
\hline Error $\varepsilon_{x_{1}}$ & 0.016 & 1.419 & 0,10935 & 0,24285 \\
\hline Error $\varepsilon_{x_{2}}$ & 0.017 & 1.479 & 0,10881 & 0,20681 \\
\hline $\min F$ & -3.996 & -3.983 & $-3,99999$ & $-3,99998$ \\
\hline
\end{tabular}

\section{Conclusion}

In this study, we have compared a method of variations with successive approximation method. By comparison of the numerical results of the methods with the exact solution the performance of the methods have been confirmed.

\section{References}

[1] Igor Grigoryev, Svetlana Mustafina, Global optimization of functions of several variables using parallel technologies International Journal of Pure and Applied Mathematics, 106, No. 1 (2016), 301-306, doi: 10.12732/ijpam.v106i1.24.

[2] Igor Grigoryev, Eldar Miftakhov, Svetlana Mustafina, Mathematical Modelling of the Copolymerization of Styrene with Maleic Anhydride in a Homogeneous Environment Int. J. Chem. Sci., 14, No. 1 (2016), 381-386. 
